\title{
EFFECT OF ANNEALING ON FREE-VOLUME PROPERTIES OF POLY(ETHYLENE TEREPHTHALATE) STUDIED BY POSITRON ANNIHILATION
}

\author{
M. Misheva ${ }^{a}$, N. Djourelov ${ }^{b}$ and I. Seganov ${ }^{c}$ \\ ${ }^{a}$ Faculty of Physics, Sofia University, 5 J. Bourchier Blvd., 1126 Sofia, Bulgaria \\ ${ }^{b}$ Institute for Nuclear Research and Nuclear Energy, Bulgarian Academy of Sciences \\ 72 Tzarigradsko shoosse Blvd., 1784 Sofia, Bulgaria \\ ${ }^{c}$ Faculty of Chemistry, Sofia University, 1 J. Bourchier Blvd., 1126 Sofia, Bulgaria
}

\begin{abstract}
The effect of annealing on free-volume properties of poly(ethylene terephthalate) was studied using a set of samples annealed for two hours in air at eight temperatures between $80^{\circ} \mathrm{C}$ and $220^{\circ} \mathrm{C}$. The fractional free-volume in the samples was estimated from ortho-positronium lifetime and its relative intensity. The variations of fractional free-volume and of the macroscopic specific volume with annealing temperature were compared. The variation of the pore volume probability density functions with the annealing temperature was found. The interdependence between $S$ - and $W$-parameters was also discussed.
\end{abstract}

PACS numbers: $78.70 . \mathrm{Bj}$

\section{Introduction}

Considerable changes in polymer amorphous parts occur during their crystallization. These changes, especially of the free-volume structure, can be studied by positron annihilation spectroscopy (PAS) methods [1]. A considerable part of positrons $(\sim 5-30 \%)$, getting into a polymer, can form a bound state with a medium electron-positronium (Ps). Positronium has two states - a singlet one, para-Ps ( $p-\mathrm{Ps})$, and a triplet one, ortho-Ps (o-Ps). The self-annihilation lifetime of $p$-Ps is about $120 \mathrm{ps}$, while $o$-Ps lives for $\sim 140 \mathrm{~ns}$ in vacuum. In condensed matter $o$-Ps tends to localize in low-density parts of the sample under study, and his lifetime $\tau$ reduces to several nanoseconds. This is caused by $o$-Ps positron annihilation with one electron, picked off from the surrounding matter. The lifetime of $o$-Ps, annihilating by pick-off process, depends on the size $R$ of the free-volume hole, at which o-Ps is trapped. The intensity of the o-Ps component in the annihilation spectra most often is connected with the number of free-volume holes $[2,3]$. 
The purpose of this study is to elucidate the changes, as seen by positrons, in the amorphous parts of poly(ethylene terephthalate) (PET), due to isochronal annealing at temperatures bellow the melting temperature. Positron lifetime (PL) and Doppler broadening of the annihilation gamma-line (DBAL) measurements were used.

\section{Experimental}

The PET samples are from Jambol, Bulgaria. Initially they are fully amorphous as was shown by wide angle X-ray scattering (WAXS). A set of nine pair of samples was used. Except for the reference pair, the samples were annealed for two hours in an oil bath in air. The annealing temperatures and pycnometric densities of the samples are shown in the Table.

TABLE

Annealing temperatures and densities of the PET samples studied.

\begin{tabular}{c|c|l|c|c|c|c|c|c|c}
\hline \hline Sample & 1 & 2 & 3 & 4 & 5 & 6 & 7 & 8 & 9 \\
\hline$T_{\mathrm{a}}\left[{ }^{\circ} \mathrm{C}\right]$ & unannealed & 80 & 100 & 120 & 140 & 160 & 180 & 200 & 220 \\
\hline$\rho \pm 0.02\left[\mathrm{~g} / \mathrm{cm}^{3}\right]$ & 1.14 & 1.14 & 1.17 & 1.32 & 1.39 & 1.45 & 1.34 & 1.45 & 1.43
\end{tabular}

The lifetime spectrometer was a standard fast-fast coincidence apparatus. It provides a time resolution $\sim 260 \mathrm{ps}$ full width at half maximum (FWHM). A positron source, ${ }^{22} \mathrm{NaCl}$ sealed with Kapton foils, was sandwiched between two identical samples. Corrections for positrons annihilating in the source were made according to [4]. The experimental spectra were fitted by the program POSITRONFIT-EXTENDED [5]. The program CONTIN (PALS-2) [6, 7] was also used. In the latter case the lifetime spectrum of well-annealed and chemically polished $\mathrm{Cu}$ samples with $\tau=121 \mathrm{ps}(>99 \%)$ was used as a reference spectrum.

The Doppler broadening of the $511 \mathrm{keV}$ annihilation $\gamma$-line was measured using a high purity germanium detector of energy resolution $1.17 \mathrm{keV}$ at $514 \mathrm{keV}$ $\gamma$-line of ${ }^{85} \mathrm{Sr}$. The annihilation $\gamma$-line is characterized by ordinary $S$-and $W$ - shape parameters.

\section{Results and discussion}

\subsection{Lifetime measurements}

The best values of $\chi_{2}$ were obtained for unrestricted three-component decomposition of the measured lifetime spectra. In general, our results agree with those of the other authors [8-10] except for the higher value of the longest-lived component, ranging from about 17 to $22 \%$. As it is commonly accepted, this component is due to the pick-off annihilation of $o$-Ps, localized in free-volume holes, and is the only one carrying the structural information. 
Assuming an infinite spherical potential of the radius $R$ with a uniform electron layer of thickness $R_{0}$, the following semi-empirical formula has been proposed $[11,12]$

$$
\tau=\left\{\lambda_{\mathrm{B}}\left[1-\frac{R}{R+R_{0}}+\frac{1}{2 \pi} \sin \left(2 \pi \frac{R}{R+R_{0}}\right)\right]\right\}^{-1},
$$

where $\lambda_{\mathrm{B}}=2 \mathrm{~ns}^{-1}$ is the spin averaged Ps annihilation rate, and $R_{0}=1.656 \AA$. The lifetime $\tau$ and the radius $R$ are expressed in ns and $\AA$, respectively.

In Refs. [2, 3, 13], it was suggested that the fractional free-volume in a sample can be expressed by the following formula:

$$
F_{v}=C V_{f} I
$$

where $I[\%]$ is the intensity of o-Ps lifetime component $\tau, V_{\mathrm{f}}$ in $\AA^{3}$ is the pore volume $-V_{f}=(4 / 3) \pi R^{3}$ and $C$ is an empirical constant that has to be obtained in a study different from the positron annihilation experiment. Obviously the parameter $V=I R^{3}$, where the pore radius $R$ is calculated by Eq. (1) from the values of $\tau$, can be used as a quantity proportional to the fractional free-volume. The dependence of $V$ on the annealing temperature is depicted in Fig. 1. In the same figure, the specific volume $V_{\mathrm{sp}}=1 / \rho$ is also presented. The same behavior of the two quantities versus annealing temperature means, in our opinion, that in the present case, the fractional free-volume depends not only on $V_{\mathrm{f}}$ but on $I_{3}$, as well. As shown by Dlubek et al. [14], this is not valid in general.

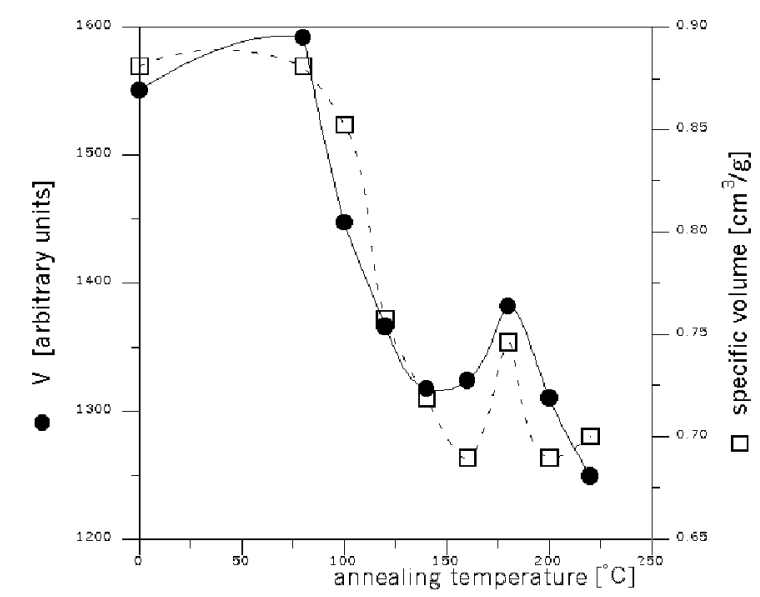

Fig. 1. Fractional free-volume parameter $V$ and specific volume $V_{\text {sp }}$ versus annealing temperature.

In Fig. 2 some representative probability density functions (PDFs) of the hole-volume, obtained from the o-Ps annihilation rate PDF (CONTIN program), are presented. Transformation formulae from [6] were used. It is interesting to note the different FWHM of the volume distributions. From the figure one can see that the isochronal annealing of PET samples at elevated temperatures leads to an increase in the FWHM of the hole-volume distributions. This means that 


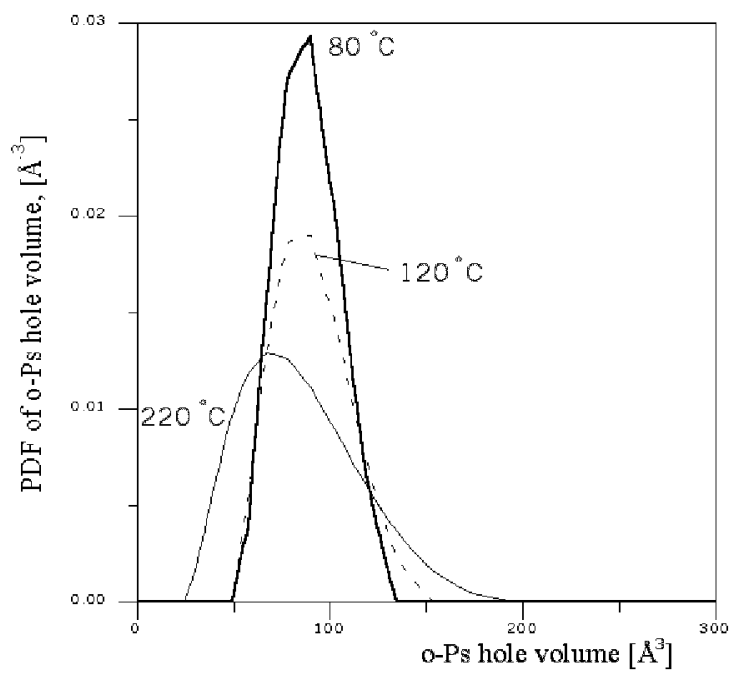

Fig. 2. Representative hole-volume distributions.

the disordering of the amorphous parts of the samples increases with an increases in the annealing temperature. This is accompanied, as it can be expected, by the decreasing of the amount of the amorphous part of the samples (area under distribution curves).

\section{2. Doppler broadening of the annihilation line measurements}

The variations of $S$-parameter and of mean positron lifetime $\tau_{\mathrm{m}}\left(\tau_{\mathrm{m}}=\right.$ $\left.\sum_{i=1}^{3} I_{i} \tau_{i}\right)$ with annealing temperature are presented in Fig. 3 . It can be seen that $S$ and $\tau_{\mathrm{m}}$, although similar, are not proportional. The latter might occur if the variations are caused by a single defect with different concentrations, which

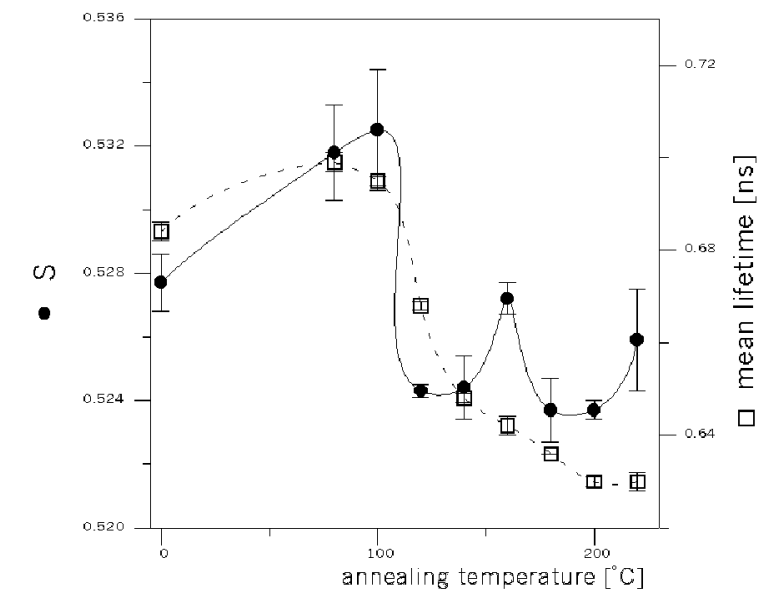

Fig. 3. S-parameter and mean positron lifetime versus annealing temperature. 
obviously is not the case. This is consistent with the observed not linear interdependence between $S$ - and $W$-parameters. The latter means [15] that the defect structure of the PET samples, isochronaly annealed at different temperatures, is different.

\section{Conclusions}

Positron lifetimes and Doppler broadening of the annihilation line measurements are applied to study the changes of the free-volume properties of the amorphous parts of poly(ethylene terephthalate) after isochronal annealing. It was established that good agreement exists between the behavior of the fractional free-volume and the specific volume versus annealing temperature. The defect structure of the samples, annealed at different temperatures, is different. The width of the hole-volume distributions increases with an increase in the annealing temperature.

\section{Acknowledgment}

This work was supported by the National Fund for Scientific Investigations (NFSI) under contract F645.

\section{References}

[1] Y.C. Jean, Mater. Sci. Forum 175-178, 59 (1995).

[2] Y.Y. Wang, H. Nakanishi, Y.C. Jean, T. Sandreczki, J. Pol. Sci. B 28, 1431 (1990).

[3] Y. Kobayashi, W. Zheng, E.F. Meyer, J.D. McGervey, A.M. Jamieson, R. Simha, Macromol. 22, 2302 (1989).

[4] N. Djourelov, M. Misheva, J. Phys., Condens. Matter 8, 2081 (1996).

[5] P. Kirkegaard, M. Eldrup, O.E. Mogensen, N.J. Pedersen, Comput. Phys. Commun. 23, 307 (1981).

[6] R. Gregory, J. Appl. Phys. 70, 4665 (1991).

[7] R. Gregory, Y. Zhu, Nucl. Instrum. Methods A 290, 172 (1990).

[8] L. Xie, D.W. Gidley, H.A. Hristov, A.F. Yee, Polymer 35, 14 (1994).

[9] H.F.M. Mohamed, Y. Ito, M. Imai, J. Chem. Phys. 105, 4841 (1996).

[10] B. Wang, C.Q. He, J.M. Zhang, S.Q. Li, S.J. Wang, J.Z. Shi, D.Z. Ma, Phys. Lett. A 235, 557 (1997).

[11] M. Eldrup, D. Lightbody, J.N. Sherood, Chem. Phys. 63, 51 (1981).

[12] H. Nakanishi, S.J. Wang, Y.C. Jean, in: Positron Annihilation Study of Fluids, Ed. S.C. Sharma, World Scientific, Singapore 1987, p. 292.

[13] H. Nakanishi, Y.Y. Wang, Y.C. Jean, T.C. Sandreczki, in: Positron Annihilation in Fluids, Ed. S.C. Sharma, World Scientific, Singapore 1987, p. 285.

[14] G. Dlubek, J. Stejny, M.A. Alam, Macromol. 31, 4574 (1998).

[15] L. Liszkay, C. Corbel, L. Baroux, P. Hautojärvi, M. Bayhan, A.W. Brinkman, S. Tatarenko, Appl. Phys. Lett. 64, 1380 (1994). 